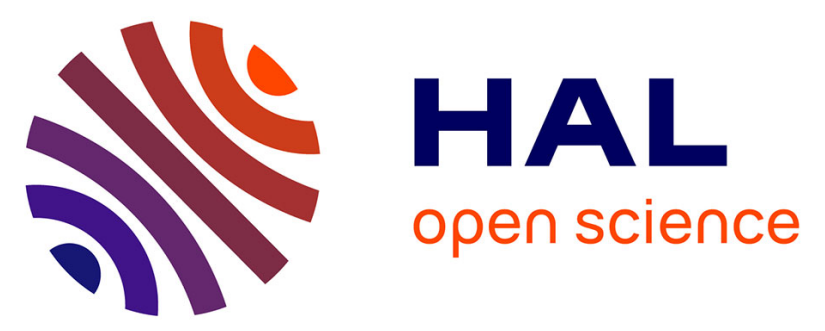

\title{
Backstepping Stabilization of 2x2 Linear Hyperbolic PDEs Coupled with Potentially Unstable Actuator and Load Dynamics
}

David Bou Saba, Federico Bribiesca Argomedo, Michaël Di Loreto, Damien Eberard

\section{To cite this version:}

David Bou Saba, Federico Bribiesca Argomedo, Michaël Di Loreto, Damien Eberard. Backstepping Stabilization of 2x2 Linear Hyperbolic PDEs Coupled with Potentially Unstable Actuator and Load Dynamics. 56th IEEE CDC, Dec 2017, Melbourne, Australia. pp.2498-2503, 10.1109/CDC.2017.8264016 . hal-01644149

\author{
HAL Id: hal-01644149 \\ https://hal.science/hal-01644149
}

Submitted on 2 Apr 2019

HAL is a multi-disciplinary open access archive for the deposit and dissemination of scientific research documents, whether they are published or not. The documents may come from teaching and research institutions in France or abroad, or from public or private research centers.
L'archive ouverte pluridisciplinaire HAL, est destinée au dépôt et à la diffusion de documents scientifiques de niveau recherche, publiés ou non, émanant des établissements d'enseignement et de recherche français ou étrangers, des laboratoires publics ou privés. 


\title{
Backstepping Stabilization of $2 \times 2$ Linear Hyperbolic PDEs Coupled with Potentially Unstable Actuator and Load Dynamics
}

\author{
David Bou Saba ${ }^{1}$, Federico Bribiesca-Argomedo ${ }^{1}$, Michael Di Loreto ${ }^{1}$ and Damien Eberard ${ }^{1}$
}

\begin{abstract}
We consider the problem of full-state feedback stabilization of a (possibly unstable) system of hyperbolic partial differential equations (PDEs). Unlike previous works, boundary couplings to linear ordinary differential equations (ODEs) at both boundaries are considered and actuation is available through one of these ODE dynamics. This structure can arise when considering linear (or linearized) systems of balance laws with finite-dimensional actuator and load dynamics. The feedback law proposed in this paper is constructed using an invertible transform based on the (infinite-dimensional) backstepping method.
\end{abstract}

\section{INTRODUCTION}

Control of systems modeled by partial differential equations (PDEs), and in particular, of hyperbolic PDEs is a very active research topic, and therefore many different approaches to tackle the stabilization and observation problems exist, see for instance the book [1].

One of these approaches, infinite-dimensional backstepping, has proven since its introduction in [2], a very successful method to construct full-state feedback gains for boundary stabilization of large classes of PDEs. It can be applied to single PDE, as illustrated by the many examples given in [3], or to systems of PDEs, such as [4] in the case of $2 \times 2$ first order linear hyperbolic systems (extended in [5] to the quasilinear case). Other relevant results concerning systems of hyperbolic PDEs are the trajectory generation and tracking problem considered in [6], and the construction of a minimum-time control provided in [7].

Many recent results in the backstepping literature have been focused on systems of PDEs coupled in some way to ODE dynamics. One may cite for example [8], where a cascade interconnection is considered between a parabolic PDE and an ODE, and [9], where a fully interconnected structure is considered instead. In the hyperbolic case, [10] considered the problem of disturbance rejection for a hyperbolic PDEODE system (with a cascade structure) and the associated observation problem in [11]. For fully interconnected structures in the hyperbolic case, [12] recently tackled the stabilization problem for heterodirectional hyperbolic PDEs coupled to an ODE.

The originality of this paper is that we apply the backstepping method to the stabilization of a first order $2 \times 2$ linear hyperbolic PDE, coupled to two linear ODEs (at both ends) but with actuation available only through one of these ODEs. For this class of systems, the results in [12] fail because the

\footnotetext{
${ }^{1}$ Université de Lyon - Laboratoire Ampère (CNRS UMR5005) - INSA de Lyon, 69621 Villeurbanne CEDEX, France. \{david.bou-saba, federico.bribiesca, michael.di-loreto, damien.eberard\} @insa-lyon. fr
}

actuation is not directly available at the PDE boundary. This structure could arise, for instance, when considering a power transmission line with a dynamic load at one boundary and actuator dynamics at the other. Other applications can be found in oil well drilling [10] and incompressible flows [13], if actuator dynamics need to be considered.

The paper is structured as follows: in Section II we present the stabilization problem under consideration. In Section III we present the backstepping design and the main stabilization result. Simulation results are shown in Section IV. At last, conclusions and perspectives are discussed in Section V.

Throughout this paper we will use the following notation for two triangular domains in $\mathbb{R}^{2}$

$$
\begin{aligned}
& \mathcal{T}_{1} \doteq\{(x, y) \in[0,1] \times[0,1], y \leq x\} \\
& \mathcal{T}_{2} \doteq\{(x, y) \in[0,1] \times[0,1], y \geq x\}
\end{aligned}
$$

Furthermore, given a set $\Omega \subseteq \mathbb{R}^{2}$, its characteristic function is denoted

$$
\mathbb{1}_{\{\Omega\}}(x, y) \doteq \begin{cases}1 & \text { if }(x, y) \in \Omega \\ 0 & \text { otherwise }\end{cases}
$$

The euclidean norm of a vector $\varphi \in \mathbb{R}^{r}, r \in \mathbb{N} \backslash\{0\}$ is denoted by $\|\varphi\|_{\mathbb{R}^{r}} \doteq\left(\varphi^{\top} \varphi\right)^{1 / 2}$. For a function in $\phi \in$ $L^{2}([0,1] ; \mathbb{R})$, its norm is defined in the usual sense

$$
\|\phi\|_{L^{2}([0,1] ; \mathbb{R})}=\left(\int_{0}^{1} \phi^{2}(s) \mathrm{d} s\right)^{1 / 2} .
$$

The notation $\mathbb{I}_{r}$ will represent the $r \times r$ identity matrix. Finally, for a symmetric matrix $A \in \mathbb{R}^{r \times r}, A \succ 0(A \prec 0)$ will indicate that $A$ is positive definite (negative definite, respectively), while $\lambda_{\min }(A)$ and $\lambda_{\max }(A)$ will stand for the smallest and the largest eigenvalues of $A$.

\section{Problem Statement}

We are concerned with the stabilization of the zero equilibrium of a class of systems described by coupled ODE-PDEODE systems. A schematic representation of such systems is depicted in Fig. 1. The thick arrows represent in-domain couplings of the PDE states, while the thin arrows represent couplings at the boundaries of the PDEs. It is worth noticing that the couplings between the PDEs and the ODEs at both ends are bidirectional, which is why existing backstepping designs for related systems (such as [10], [4], or [12]) cannot be applied. 


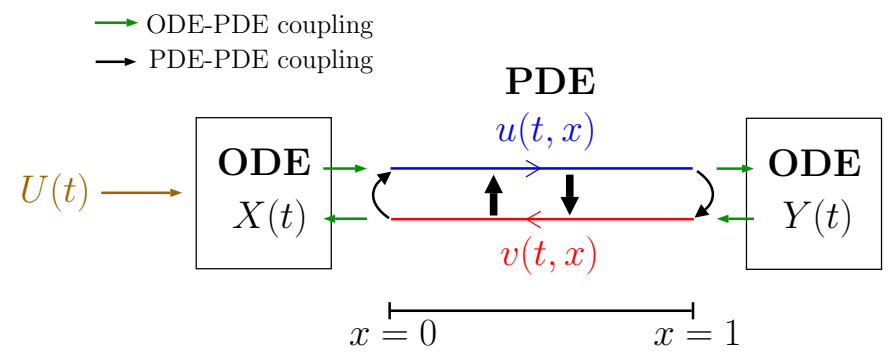

Fig. 1: Schematic representation of the coupled ODE-PDE-ODE system (2)

More precisely, we consider systems of the form:

$$
\begin{aligned}
u_{t}(t, x) & =-\lambda u_{x}(t, x)+\sigma^{++} u(t, x)+\sigma^{+-} v(t, x) \\
v_{t}(t, x) & =\lambda v_{x}(t, x)+\sigma^{-+} u(t, x)+\sigma^{--} v(t, x) \\
\dot{X}(t) & =A_{0} X(t)+E_{0} v(t, 0)+B_{0} U(t) \\
\dot{Y}(t) & =A_{1} Y(t)+E_{1} u(t, 1) \\
u(t, 0) & =R_{0} v(t, 0)+C_{0} X(t) \\
v(t, 1) & =R_{1} u(t, 1)+C_{1} Y(t)
\end{aligned}
$$

for all $t \in[0, T], x \in[0,1]$, where $\lambda>0$ is the transport speed associated to the two transport equations and $\sigma^{i j} \in \mathbb{R}(i, j \in\{+,-\})$ represent constant in-domain couplings between the PDEs. The matrices appearing in (2c)(2f) are real-valued with appropriate size and $U(t) \in \mathbb{R}^{n}$ is the control input. The associated initial conditions are $X(0) \in \mathbb{R}^{n}, Y(0) \in \mathbb{R}^{m}$ and $u(0, \cdot), v(0, \cdot)$ belonging to suitable functional spaces (cf. subsection III-D) and potentially satisfying adequate compatibility conditions (according to the required regularity of solutions).

The following assumptions are made in this paper:

Assumption 1: $B_{0}$ is invertible (this implies in particular that the pair $\left(A_{0}, B_{0}\right)$ is controllable).

Assumption 2: $\operatorname{rank}\left(C_{0}\right) \geq 1$.

Assumption 3: $R_{1} \neq 0$.

Assumption 4: The pair $\left(A_{1}, E_{1}\right)$ is stabilizable (i.e. there exists $F_{1} \in \mathbb{R}^{1 \times m}$ such that the matrix $A_{1}+E_{1} F_{1}$ is Hurwitz).

Notice that the precise sense given to equation (2) depends on the type of solutions considered (weak vs. classical), the choice of functional spaces for the states of the system, and the initial conditions. In order to keep this paper concise and easily readable, we will impose strong conditions to the system (and its initial conditions) in order to deal only with classical solutions. Extensions to more general cases are outside the scope of the present work.

\section{BACKSTEPPING CONTROL Design}

\section{A. Transform and Target System}

In order to construct a stabilizing control law, we will employ the backstepping procedure. Therefore, we will look for $\longrightarrow$ ODE-PDE coupling

E coupling

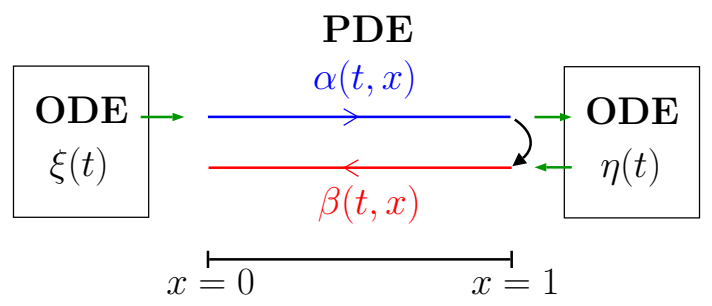

Fig. 2: Schematic representation of the target system (4)

a linear (bounded and boundedly invertible) transformation

$$
\begin{aligned}
\alpha(t, x)= & u(t, x)-\int_{x}^{1} P^{11}(x, y) u(t, y) \mathrm{d} y \\
& -\int_{x}^{1} P^{12}(x, y) v(t, y) \mathrm{d} y-g(x) Y(t) \\
\beta(t, x)= & v(t, x)-\int_{x}^{1} P^{21}(x, y) u(t, y) \mathrm{d} y \\
& -\int_{x}^{1} P^{22}(x, y) v(t, y) \mathrm{d} y-\gamma(x) Y(t) \\
\xi(t)= & X(t)-D Y(t)-q v(t, 0) \\
& -\int_{0}^{1} p_{1}(y) u(t, y) \mathrm{d} y-\int_{0}^{1} p_{2}(y) v(t, y) \mathrm{d} y \\
\eta(t)= & Y(t)
\end{aligned}
$$

that transforms system (2) into the (stable) target system

$$
\begin{aligned}
\alpha_{t}(t, x) & =-\lambda \alpha_{x}(t, x)+\sigma^{++} \alpha(t, x) \\
\beta_{t}(t, x) & =\lambda \beta_{x}(t, x)+\sigma^{--} \beta(t, x) \\
\dot{\xi}(t) & =\left(A_{0}+B_{0} F_{0}\right) \xi(t) \\
\dot{\eta}(t) & =\left(A_{1}+E_{1} F_{1}\right) \eta(t)+E_{1} \alpha(t, 1) \\
\alpha(t, 0) & =C_{0} \xi(t) \\
\beta(t, 1) & =R_{1} \alpha(t, 1)+C_{1} \eta(t)
\end{aligned}
$$

where $F_{0} \in \mathbb{R}^{n \times n}$ and $F_{1} \in \mathbb{R}^{1 \times m}$ are chosen such that the matrices $\left(A_{0}+B_{0} F_{0}\right)$ and $\left(A_{1}+E_{1} F_{1}\right)$ are Hurwitz. $\xi_{0}=\xi(0)$ and $\eta_{0}=\eta(0)$ are respectively the initial conditions for ODE states $\xi(t)$ and $\eta(t)$, and $\alpha_{0}(x)=\alpha(0, x), \beta_{0}(x)=\beta(0, x)$ are the initial conditions for the PDE states $\alpha(t, x)$ and $\beta(t, x)$ respectively. A schematic representation of the target system (4) is given in Fig. 2. This structure was chosen since it eliminates all couplings that can be a source of instability.

\section{B. Properties of the Target System}

Before finding transform (3) we will state some properties of the target system that will be required throughout the paper. 
In order to define a precise sense of solutions to the systems considered in this paper we will introduce a suitable space.

Definition 1: We define the space $\Xi \doteq C^{1}([0,1] ; \mathbb{R}) \times$ $C^{1}([0,1] ; \mathbb{R}) \times \mathbb{R}^{n} \times \mathbb{R}^{m}$ equipped with the norm

$$
\begin{aligned}
& \|\Psi\|_{\Xi} \doteq \max \left\{\left\|\Psi_{1}\right\|_{C^{1}([0,1] ; \mathbb{R})},\left\|\Psi_{2}\right\|_{C^{1}([0,1] ; \mathbb{R})}\right. \\
& \left.\left\|\Psi_{3}\right\|_{\mathbb{R}^{n}},\left\|\Psi_{4}\right\|_{\mathbb{R}^{m}}\right\}
\end{aligned}
$$

for $\Psi=\left(\Psi_{1}, \Psi_{2}, \Psi_{3}, \Psi_{4}\right) \in \Xi$, where $\|\cdot\|_{C^{1}([0,1] ; \mathbb{R})}$ is the usual norm in $C^{1}([0,1] ; \mathbb{R})$

$$
\|\phi\|_{C^{1}([0,1] ; \mathbb{R})} \doteq \sup _{x \in[0,1]}|\phi(x)|+\sup _{x \in[0,1]}\left|\phi^{\prime}(x)\right| .
$$

Remark that $\left(\Xi,\|\cdot\|_{\Xi}\right)$ is a Banach space.

Lemma 1: Given an initial condition $(\alpha(0, \cdot), \beta(0, \cdot), \xi(0), \eta(0)) \in \Xi$ verifying the compatibility conditions

$$
\begin{aligned}
\alpha(0,0)= & C_{0} \xi(0) \\
\beta(0,1)= & R_{1} \alpha(0,1)+C_{1} \eta(0) \\
\alpha_{x}(0,0)= & \frac{\sigma^{++}}{\lambda} \alpha(0,0)-\frac{1}{\lambda} C_{0}\left(A_{0}+B_{0} F_{0}\right) \xi(0) \\
\beta_{x}(0,1)= & -\frac{\sigma^{--}}{\lambda} \beta(0,1)+\frac{1}{\lambda}\left(\sigma^{++} R_{1}+C_{1} E_{1}\right) \alpha(0,1) \\
& -R_{1} \alpha_{x}(0,1)+\frac{1}{\lambda} C_{1}\left(A_{1}+E_{1} F_{1}\right) \eta(0)
\end{aligned}
$$

system (4) has a unique (classical) solution $(\alpha(t, \cdot), \beta(t, \cdot), \xi(t), \eta(t)) \in \Xi$ for all $t \in[0, T]$ satisfying $\xi(\cdot) \in C^{\infty}\left([0, T] ; \mathbb{R}^{n}\right), \eta(\cdot) \in C^{2}\left([0, T] ; \mathbb{R}^{m}\right), \alpha(\cdot, \cdot)$, $\beta(\cdot, \cdot) \in C^{1}([0, T] \times[0,1] ; \mathbb{R})$.

Due to space constraints, the proof of this result is not included here. However, one should note that the compatibility conditions required for a $C^{1}$-solution are not at all surprising, see for instance the analogous conditions in [5] (for an $H^{2}$ solution). In this paper we will only consider these solutions and will not seek to remove the (strong) compatibility condition.

In order to define a sense for the stability of system (4) we will introduce a space containing all the components of the system and define a suitable norm in this space.

Definition 2: We define the space $\chi \doteq L^{2}([0,1] ; \mathbb{R}) \times$ $L^{2}([0,1] ; \mathbb{R}) \times \mathbb{R}^{n} \times \mathbb{R}^{m}$ equipped with the norm

$$
\begin{aligned}
& \|\Psi\|_{\chi} \doteq\left(\left\|\Psi_{1}\right\|_{L^{2}([0,1] ; \mathbb{R})}^{2}+\left\|\Psi_{2}\right\|_{L^{2}([0,1] ; \mathbb{R})}^{2}\right. \\
& \left.\quad+\left\|\Psi_{3}\right\|_{\mathbb{R}^{n}}^{2}+\left\|\Psi_{4}\right\|_{\mathbb{R}^{m}}^{2}\right)^{1 / 2}
\end{aligned}
$$

for $\Psi=\left(\Psi_{1}, \Psi_{2}, \Psi_{3}, \Psi_{4}\right) \in \chi$.

Remark that $\left(\chi,\|\cdot\|_{\chi}\right)$ is a Banach space.

Lemma 2: The zero equilibrium (origin) of system (4) is exponentially stable in the $\chi$-norm. There exist real constants $M, \omega>0$ such that, for all initial conditions $(\alpha(0, \cdot), \beta(0, \cdot), \xi(0), \eta(0)) \in \chi$ satisfying the conditions of Lemma 1 the solution $\Psi(t) \doteq(\alpha(t, \cdot), \beta(t, \cdot), \xi(t), \eta(t))$ of system (4) satisfies

$$
\|\Psi(t)\|_{\chi} \leq M e^{-\omega t}\|\Psi(0)\|_{\chi}, \quad \forall t \in[0, T] .
$$

\section{Properties of the Backstepping Transform}

Now we state some properties of the transform defined in (3).

Lemma 3: Given $P^{i j} \in C^{1}\left(\mathcal{T}_{2} ; \mathbb{R}\right), i, j \in\{1,2\}, g, \gamma \in$ $C^{1}\left([0,1] ; \mathbb{R}^{m}\right)$ and $p_{1}, p_{2} \in C^{1}\left([0,1] ; \mathbb{R}^{n}\right)$ the backstepping transform defined in (3) is a bounded linear operator from $\chi$ to $\chi$ (alternatively from $\Xi$ to $\Xi$ ). Furthermore, it has a bounded inverse in $\chi$ (in $\Xi$ respectively).

The boundedness is clear from equation (3). Invertibility follows in turn from the structure of the proposed transform together with the invertibility of Volterra integral equations of the second kind (or the zero spectral radius property of Volterra integral operators) [14, Theorem 3.10].

Lemma 4: A sufficient condition for the backstepping transform (3) to (invertibly) map a classical solution of system (2) into a corresponding one of system (4) is that the kernels $P^{i j}, i, j \in\{1,2\}, p_{1}, p_{2}$, the coefficients $g, \gamma$, $D$ and $q$, and the control input $U(t)$ satisfy the regularity conditions of Lemma 3 as well as the following equations

$$
\begin{aligned}
& P_{x}^{11}(x, y)+P_{y}^{11}(x, y)=-\frac{\sigma^{-+}}{\lambda} P^{12}(x, y) \\
& P_{x}^{12}(x, y)-P_{y}^{12}(x, y)= \frac{\sigma^{++}-\sigma^{--}}{\lambda} P^{12}(x, y) \\
&-\frac{\sigma^{+-}}{\lambda} P^{11}(x, y) \\
& P_{x}^{21}(x, y)-P_{y}^{21}(x, y)= \frac{\sigma^{++}-\sigma^{--}}{\lambda} P^{21}(x, y) \\
&+\frac{\sigma^{-+}}{\lambda} P^{22}(x, y) \\
& P_{x}^{22}(x, y)+P_{y}^{22}(x, y)= \frac{\sigma^{+-}}{\lambda} P^{21}(x, y) \\
& g^{\prime}(x)=\frac{1}{\lambda} g(x)\left[\sigma^{++} \mathbb{I}_{m}\right.\left.-A_{1}\right]-P^{12}(x, 1) C_{1} \\
& \gamma^{\prime}(x)=\frac{1}{\lambda} \gamma(x)\left[-\sigma^{--} \mathbb{I}_{m}+A_{1}\right]+P^{22}(x, 1) C_{1}
\end{aligned}
$$

with boundary (final) conditions

$$
\begin{aligned}
P^{11}(x, 1) & =P^{12}(x, 1) R_{1}+\frac{1}{\lambda} g(x) E_{1} \\
P^{12}(x, x) & =-\frac{\sigma^{+-}}{2 \lambda}, \quad P^{21}(x, x)=\frac{\sigma^{-+}}{2 \lambda} \\
P^{22}(x, 1) & =\frac{1}{R_{1}} P^{21}(x, 1)-\frac{1}{\lambda R_{1}} \gamma(x) E_{1} \\
g(1) & =F_{1}, \quad \gamma(1)=R_{1} F_{1}
\end{aligned}
$$

and algebraic conditions

$$
\begin{aligned}
C_{0} D & =g(0) \\
C_{0} q & =-R_{0} \\
C_{0} p_{1}(y) & =P^{11}(0, y) \\
C_{0} p_{2}(y) & =P^{12}(0, y)
\end{aligned}
$$

with the control given by

$$
\begin{aligned}
U(t)= & k_{1} v(t, 0)+k_{2} v_{t}(t, 0)+k_{3} u(t, 1)+k_{4} X(t) \\
& +k_{5} Y(t)+\int_{0}^{1} k_{6}(y) u(t, y) \mathrm{d} y+\int_{0}^{1} k_{7}(y) v(t, y) \mathrm{d} y
\end{aligned}
$$

The proof of this result is given in Appendix A. 
where

$$
\begin{aligned}
& k_{1} \doteq B_{0}^{-1}\left(\lambda R_{0} p_{1}(0)-\lambda p_{2}(0)-\left(A_{0}+B_{0} F_{0}\right) q-E_{0}\right) \\
& k_{2} \doteq B_{0}^{-1} q \\
& k_{3} \doteq B_{0}^{-1}\left(D E_{1}-\lambda p_{1}(1)+\lambda R_{1} p_{2}(1)\right) \\
& k_{4} \doteq F_{0}+\lambda B_{0}^{-1} p_{1}(0) C_{0} \\
& k_{5} \doteq B_{0}^{-1}\left(D A_{1}-\left(A_{0}+B_{0} F_{0}\right) D+\lambda p_{2}(1) C_{1}\right) \\
& k_{6}(y) \doteq B_{0}^{-1}\left(\lambda p_{1}^{\prime}(y)+\left(\sigma^{++} \mathbb{I}_{n}-A_{0}-B_{0} F_{0}\right) p_{1}(y)\right. \\
& \left.+\sigma^{-+} p_{2}(y)\right) \\
& k_{7}(y) \doteq B_{0}^{-1}\left(-\lambda p_{2}^{\prime}(y)+\left(\sigma^{--} \mathbb{I}_{n}-A_{0}-B_{0} F_{0}\right) p_{2}(y)\right. \\
& \left.+\sigma^{+-} p_{1}(y)\right) \text {. }
\end{aligned}
$$

Let us remark that we can always find a solution to the algebraic equations (12) by using the right-inverse $C_{0}^{\dagger} \doteq$ $C_{0}^{\boldsymbol{\top}}\left(C_{0} C_{0}^{\boldsymbol{\top}}\right)^{-1}$, which is well defined by Assumption 2.

The proof of this Lemma follows the standard backstepping procedure (see for instance [3]) of differentiating the transformed solution w.r.t. time and space and substituting it in the target dynamics (plus some integration by parts where necessary). A detailed derivation of the kernel equations is omitted in this paper. We will instead focus on the existence, uniqueness and regularity of solutions to the kernel PDEs and accompanying equations.

Lemma 5: Equations (10), (11) have a unique solution verifying the conditions of Lemma 3.

Proof: First, we will define a change of variables: $\bar{x} \doteq$ $1-x, \quad \bar{y} \doteq 1-y$ and the kernels $S^{i j}(\bar{x}, \bar{y}) \doteq P^{i j}(x, y)$, $i, j \in\{1,2\}$.

Furthermore, the ODE verified by $g$ in (10e) can be rewritten in a PDE form as in [12], by defining

$$
\tilde{g}(x, y) \doteq \mathbb{1}_{\{y=1\}}(x, y) g(x),
$$

where $\tilde{g}$ verifies

$$
\begin{array}{r}
\lambda \tilde{g}_{x}(x, y)=\mathbb{1}_{\{y=1\}}(x, y)\left[\tilde{g}(x, y)\left[\sigma^{++} \mathbb{I}_{m}-A_{1}\right]\right. \\
\left.-\lambda P^{12}(x, y) C_{1}\right]
\end{array}
$$

for $(x, y) \in \mathcal{T}_{2}$, with boundary condition

$$
\tilde{g}(x, x)=\mathbb{1}_{\{y=1\}}(x, x) F_{1} .
$$

We also define the corresponding PDE in the new variables

$$
h(\bar{x}, \bar{y}) \doteq \tilde{g}(x, y) .
$$

In these variables, the kernel equations are of the class considered in [12, Theorem 3.2]. Therefore we can conclude that equations (10), (11) have a unique solution $P^{i j} \in$ $L^{\infty}\left(\mathcal{T}_{2} ; \mathbb{R}\right), i, j \in\{1,2\}, g, \gamma \in C^{1}\left([0,1] ; \mathbb{R}^{m}\right)$ and $p_{1}$, $p_{2} \in C^{1}\left([0,1] ; \mathbb{R}^{n}\right)$. After the change of variables, the resulting characteristic lines corresponding to $S^{11}, S^{12}$ and $h$ are shown in Fig. 3, as well as the line required by [12, Theorem 3.2]. The rest of the kernels are decoupled from these three kernels but behave in the same way.

Following the proof of [12, Theorem 3.2] and minor adaptation using sup norm, we obtain that the kernels are continuous in their domains. Finally, the boundary conditions in (11) and (12) ensure that the kernels are $C^{1}$-functions.

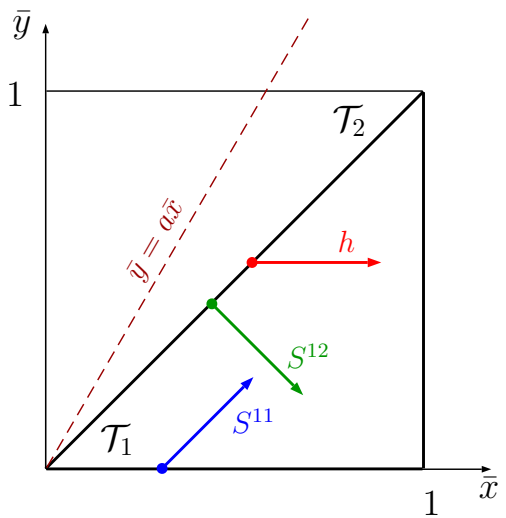

Fig. 3: Characteristic lines verified by the kernels $S^{11}(\bar{x}, \bar{y}), S^{12}(\bar{x}, \bar{y})$ and $h(\bar{x}, \bar{y})$ in $\mathcal{T}_{1}$

\section{Properties of the Closed-Loop System}

Lemma 6: Given an initial condition $(u(0, \cdot), v(0, \cdot), X(0), Y(0)) \in \Xi$ verifying the compatibility conditions

$$
\begin{aligned}
u(0,0)= & R_{0} v(0,0)+C_{0} X(0) \\
v(0,1)= & R_{1} u(0,1)+C_{1} Y(0) \\
u_{x}(0,0)= & -R_{0} v_{x}(0,0)+\frac{\sigma^{++}-R_{0} \sigma^{-+}}{\lambda} u(0,0) \\
& +\frac{1}{\lambda}\left(\sigma^{+-}-R_{0} \sigma^{--}-C_{0} E_{0}\right) v(0,0) \\
& -\frac{1}{\lambda} C_{0} A_{0} X(0)-\frac{1}{\lambda} C_{0} B_{0} U(0) \\
v_{x}(0,1)= & -R_{1} u_{x}(0,1) \\
& +\frac{1}{\lambda}\left(R_{1} \sigma^{++}-\sigma^{-+}+C_{1} E_{1}\right) u(0,1) \\
& +\frac{R_{1} \sigma^{+-}-\sigma^{--}}{\lambda} v(0,1)+\frac{1}{\lambda} C_{1} A_{1} Y(0)
\end{aligned}
$$

and $U(t)$ satisfying the conditions of Lemma 4, system (2) has a unique (classical) solution $(u(t, \cdot), v(t, \cdot), X(t), Y(t)) \in \Xi$ for all $t \in[0, T]$ satisfying $u(\cdot, \cdot), v(\cdot, \cdot) \in C^{1}([0, T] \times[0,1] ; \mathbb{R}), X(\cdot) \in C^{1}\left([0, T] ; \mathbb{R}^{n}\right)$, $Y(\cdot) \in C^{1}\left([0, T] ; \mathbb{R}^{m}\right)$.

Proof: Any initial condition of (2) in $\Xi$ is mapped, by Lemma 3 together with Lemma 5 to a bounded condition in $\Xi$ for (4). By the definition of the backstepping transform (3) and with kernels and control signal satisfying Lemma 4 (which always exist by virtue of Lemma 5), the compatibility conditions in this Lemma imply that the compatibility conditions of Lemma 1 are verified. This in turn implies that the target system (4) has a unique classical solution with adequate regularity. Using Lemma 4 and the bounded invertibility of the transform (in $\Xi$ ), from Lemma 3 and Lemma 5, we conclude that the original system (2) has a unique classical solution with the desired regularity.

We are now able to state the main result of this paper:

Theorem 7: The origin of system (2), verifying the conditions of Lemma 6 is exponentially stable in the $\chi$-norm (8). Furthermore the control law (13) verifies $U(\cdot) \in$ $C\left([0, T] ; \mathbb{R}^{n}\right)$. 
Proof: The proof follows from the fact that Lemma 6 guarantees the existence of a unique (adequately regular) classical solution to the closed-loop system with the control law defined by Lemma 4, which is linked by a bounded and boundedly invertible operator to the corresponding classical solution of Lemma 1 which satisfies the requirements for exponential stability in the sense of Lemma 2 (since $\Xi \subset$ $\chi)$. Boundedness of the inverse transform in $\chi$ space, from Lemma 3 together with Lemma 5, gives the conclusion of the theorem.

\section{Simulation Results}

The effectiveness of the control approach is illustrated in a simulation example, with $\lambda=1, \sigma^{++}=0.2$, $\sigma^{+-}=0.3, \sigma^{-+}=1, \sigma^{--}=-0.1, R_{0}=1.5$, $R_{1}=1.1, C_{0}=\left[\begin{array}{llll}1 & -1 & 2 & 3\end{array}\right], C_{1}=[1,-2,4]$, $E_{0}=\left[\begin{array}{llll}2 & -5 & 1 & -3\end{array}\right]^{\top}, \quad E_{1}=\left[\begin{array}{lll}1 & -2 & 1\end{array}\right]^{\top}$, $A_{0}=1 / 7\left[\begin{array}{cccc}1 & 4 & 3 & 0 \\ -2 & -4 & 6 & 7 \\ 4 & 9 & -5 & 5 \\ -8 & 7 & 4 & 3\end{array}\right], A_{1}=1 / 7\left[\begin{array}{ccc}2 & 1 & -4 \\ 1 & 0 & 2 \\ 3 & 7 & 8\end{array}\right]$, and $B_{0}=\left[\begin{array}{cccc}2 & 0 & 0 & 0 \\ 0 & 1 & 0 & 0 \\ 0 & 0 & -3 & 0 \\ 0 & 0 & 0 & 1\end{array}\right]$

The parameters were chosen in order to have an unstable response of system (2). The evolution of the PDE states $u(t, x)$ and $v(t, x)$ is presented in Fig. 4(a) and (b) respectively. As expected, the controller renders the closedloop system asymptotically stable.

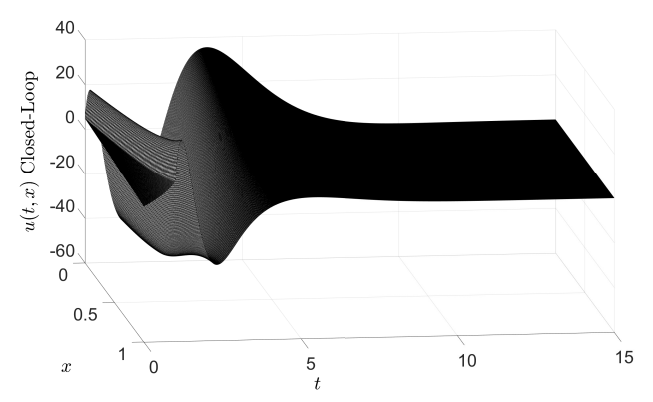

(a)

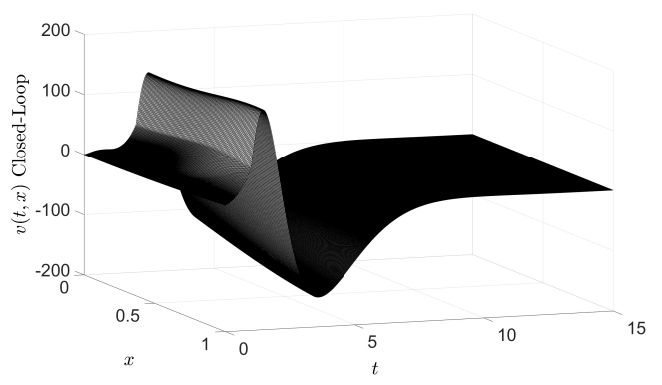

(b)

Fig. 4: Simulated evolution of the closed-loop PDE states and $\chi$-norm behaviour of the in terconnected ODE-PDE-ODE system. (a) $u(t, x)$, (b) $v(t, x)$.

\section{Conclusions And Perspectives}

In this article, the problem of stabilization of a $2 \times 2$ system of linear first-order hyperbolic PDEs coupled to linear ODEs at both ends is solved under the assumption of an invertible input matrix to one of the ODEs. The design is based on a backstepping transform and a suitable (exponentially stable) target system. The resulting control is a full-state feedback and requires knowledge of the PDE and ODE states in the whole domain, as well as the derivative with respect to time of a point in the PDE state.

Future work will be focused on designing the observer for the ODE-PDE-ODE system and avoiding the use of the timederivatives in the control. Also, the extension of these results to systems where the input matrix $\left(B_{0}\right)$ is not invertible will be explored.

\section{APPENDIX}

\section{A. Proof of Lemma 2}

Proof: [Lemma 2] We consider an extended version of the Lyapunov functional used in [12]

$$
V(t)=V_{1}(t)+V_{2}(t)+V_{3}(t)+V_{4}(t)
$$

where

$$
\begin{aligned}
& V_{1}(t)=\frac{\epsilon}{\lambda} \int_{0}^{1} e^{-\delta x} \alpha^{2}(t, x) \mathrm{d} x \\
& V_{2}(t)=\frac{1}{\lambda} \int_{0}^{1} e^{\delta x} \beta^{2}(t, x) \mathrm{d} x \\
& V_{3}(t)=\xi(t)^{\top} \Pi_{0} \xi(t) \\
& V_{4}(t)=\eta(t)^{\top} \Pi_{1} \eta(t) .
\end{aligned}
$$

The design parameters $\epsilon>0, \delta>0$ and the symmetric positive definite matrices $\Pi_{0} \in \mathbb{R}^{n \times n}$ and $\Pi_{1} \in \mathbb{R}^{m \times m}$ are yet to be determined. Let

$$
\begin{aligned}
& \kappa_{1} \doteq \min \left\{\frac{\epsilon}{\lambda} e^{-\delta}, \frac{1}{\lambda}, \lambda_{\min }\left(\Pi_{0}\right), \lambda_{\min }\left(\Pi_{1}\right)\right\} \\
& \kappa_{2} \doteq \max \left\{\frac{\epsilon}{\lambda}, \frac{e^{\delta}}{\lambda}, \lambda_{\max }\left(\Pi_{0}\right), \lambda_{\max }\left(\Pi_{1}\right)\right\} .
\end{aligned}
$$

$V(t)$ can be bounded as follows

$$
\kappa_{1}\|\Psi(t)\|_{\chi}^{2} \leq V(t) \leq \kappa_{2}\|\Psi(t)\|_{\chi}^{2}, \quad \forall t \in[0, T] .
$$

We proceed by differentiating each term of $V(t)$ along the (classical) solution of (4)

$$
\begin{aligned}
& \dot{V}_{1}(t)=2 \frac{\epsilon}{\lambda} \int_{0}^{1} e^{-\delta x} \alpha(t, x)\left[-\lambda \alpha_{x}(t, x)+\sigma^{++} \alpha(t, x)\right] \mathrm{d} x \\
& \dot{V}_{2}(t)=\frac{2}{\lambda} \int_{0}^{1} e^{\delta x} \beta(t, x)\left[\lambda \beta_{x}(t, x)+\sigma^{--} \beta(t, x)\right] \mathrm{d} x \\
& \dot{V}_{3}(t)=\xi(t)^{\top}\left[\bar{A}_{0}^{\top} \Pi_{0}+\Pi_{0} \bar{A}_{0}\right] \xi(t) \\
& \dot{V}_{4}(t)=\eta(t)^{\top}\left[\bar{A}_{1}^{\top} \Pi_{1}+\Pi_{1} \bar{A}_{1}\right] \eta(t)+2 \alpha(t, 1) E_{1}^{\top} \Pi_{1} \eta(t)
\end{aligned}
$$


where we have defined $\bar{A}_{0} \doteq A_{0}+B_{0} F_{0}$ and $\bar{A}_{1} \doteq A_{1}+$ $E_{1} F_{1}$ (Hurwitz). Integrating (24a) by parts and using (4a) and (4e), we have that

$$
\begin{aligned}
\dot{V}_{1}(t)= & -\epsilon\left[e^{-\delta x} \alpha^{2}(t, x)\right]_{x=0}^{x=1}-\delta \epsilon \int_{0}^{1} e^{-\delta x} \alpha^{2}(t, x) \mathrm{d} x \\
& +2 \frac{\epsilon \sigma^{++}}{\lambda} \int_{0}^{1} e^{-\delta x} \alpha^{2}(t, x) \mathrm{d} x \\
= & -\epsilon e^{-\delta} \alpha^{2}(t, 1)+\epsilon \xi(t)^{\top} C_{0}^{\top} C_{0} \xi(t) \\
& -\epsilon\left(\delta-2 \frac{\sigma^{++}}{\lambda}\right) \int_{0}^{1} e^{-\delta x} \alpha^{2}(t, x) \mathrm{d} x
\end{aligned}
$$

Similarly, integrating (24b) by parts and using (4b) and (4f), we have that

$$
\begin{array}{r}
\dot{V}_{2}(t) \leq \quad 2 e^{\delta} R_{1}^{2} \alpha^{2}(t, 1)+2 e^{\delta} \eta(t)^{\top} C_{1}^{\top} C_{1} \eta(t) \\
-\left(\delta-2 \frac{\sigma^{--}}{\lambda}\right) \int_{0}^{1} e^{\delta x} \beta^{2}(t, x) \mathrm{d} x .
\end{array}
$$

Applying Young's inequality for the term $2 \alpha(t, 1) E_{1}^{\top} \Pi_{1} \eta(t)$ in (24d) yields

$$
\begin{aligned}
\dot{V}_{4}(t) \leq & \eta(t)^{\top}\left[\bar{A}_{1}^{\top} \Pi_{1}+\Pi_{1} \bar{A}_{1}\right] \eta(t) \\
& +E_{1}^{\top} \Pi_{1}^{2} E_{1} \alpha^{2}(t, 1)+\eta(t)^{\top} \eta(t) .
\end{aligned}
$$

Differentiating (20) and using (24c), (25)-(27) yield

$$
\begin{aligned}
\dot{V}(t) \leq & -\epsilon\left(\delta-2 \frac{\sigma^{++}}{\lambda}\right) \int_{0}^{1} e^{-\delta x} \alpha^{2}(t, x) \mathrm{d} x \\
& -\left(\delta-2 \frac{\sigma^{--}}{\lambda}\right) \int_{0}^{1} e^{\delta x} \beta^{2}(t, x) \mathrm{d} x \\
& -\left[\epsilon e^{-\delta}-2 e^{\delta} R_{1}^{2}-E_{1}^{\top} \Pi_{1}^{2} E_{1}\right] \alpha^{2}(t, 1) \\
& +\xi(t)^{\boldsymbol{\top}}\left[\bar{A}_{0}^{\top} \Pi_{0}+\Pi_{0} \bar{A}_{0}+\epsilon C_{0}^{\top} C_{0}\right] \xi(t) \\
& +\eta(t)^{\boldsymbol{\top}}\left[\bar{A}_{1}^{\top} \Pi_{1}+\Pi_{1} \bar{A}_{1}+2 e^{\delta} C_{1}^{\top} C_{1}+\mathbb{I}_{m}\right] \eta(t) .
\end{aligned}
$$

The design parameters $\delta, \Pi_{1}, \epsilon$ and $\Pi_{0}$ can always be chosen such that the following inequalities are verified:

$$
\begin{aligned}
& \delta>\frac{2}{\lambda} \max \left\{\sigma^{++}, \sigma^{--}\right\} \\
& \bar{A}_{1}^{\top} \Pi_{1}+\Pi_{1} \bar{A}_{1}+2 e^{\delta} C_{1}^{\top} C_{1}+\mathbb{I}_{m} \prec 0 \\
& \epsilon>2 e^{2 \delta} R_{1}^{2}+e^{\delta} E_{1}^{\top} \Pi_{1}^{2} E_{1} \\
& \bar{A}_{0}^{\top} \Pi_{0}+\Pi_{0} \bar{A}_{0}+\epsilon C_{0}^{\top} C_{0} \prec 0 .
\end{aligned}
$$

Taking

$$
\begin{aligned}
\omega=\frac{1}{2} \min \{ & \lambda \delta-2 \sigma^{++}, \lambda \delta-2 \sigma^{--}, \\
& -\frac{\lambda_{\max }\left(\bar{A}_{0}^{\top} \Pi_{0}+\Pi_{0} \bar{A}_{0}+\epsilon C_{0}^{\top} C_{0}\right)}{\lambda_{\max }\left(\Pi_{0}\right)}, \\
& \left.-\frac{\lambda_{\max }\left(\bar{A}_{1}^{\top} \Pi_{1}+\Pi_{1} \bar{A}_{1}+\Pi_{1}+2 e^{\delta} C_{1}^{\top} C_{1}\right)}{\lambda_{\max }\left(\Pi_{1}\right)}\right\}
\end{aligned}
$$

we have that

$$
\dot{V}(t) \leq-2 \omega V(t) .
$$

Finally, integrating (31), we have

$$
V(t) \leq \mathrm{e}^{-2 \omega t} V(0),
$$

and using (23), we get

$$
\|\Psi(t)\|_{\chi} \leq\left(\frac{\kappa_{2}}{\kappa_{1}}\right)^{1 / 2} e^{-\omega t}\|\Psi(0)\|_{\chi}, \quad \forall t \in[0, T] .
$$

\section{REFERENCES}

[1] G. Bastin and J.-M. Coron, Stability and boundary stabilization of 1-D hyperbolic systems, ser. Progress in Nonlinear Differential Equations and Their Applications Subseries in Control. Birkhäuser, 2016, vol. 88 .

[2] A. Balogh and M. Krstic, "Infinite dimensional backstepping-style feedback transformations for a heat equation with an arbitrary level of instability," European Journal of Control, vol. 8, no. 2, pp. 165-175, 2002.

[3] M. Krstic and A. Smyshlyaev, Boundary control of PDEs: A course on backstepping designs. SIAM, 2008, vol. 16.

[4] R. Vazquez, M. Krstic, and J.-M. Coron, "Backstepping boundary stabilization and state estimation of a $2 \times 2$ linear hyperbolic system," in Proceedings of the 50th IEEE Conference on Decision and Control and European Control Conference. IEEE, 2011, pp. 4937-4942.

[5] J.-M. Coron, R. Vazquez, M. Krstic, and G. Bastin, "Local exponential $H^{2}$ stabilization of a $2 \times 2$ quasilinear hyperbolic system using backstepping," SIAM Journal on Control and Optimization, vol. 51, no. 3, pp. 2005-2035, 2013.

[6] P.-O. Lamare and N. Bekiaris-Liberis, "Control of $2 \times 2$ linear hyperbolic systems: Backstepping-based trajectory generation and PI-based tracking," Systems \& Control Letters, vol. 86, pp. 24-33, 2015.

[7] J. Auriol and F. Di Meglio, "Minimum time control of heterodirectional linear coupled hyperbolic PDEs," Automatica, vol. 71, pp. 300307, 2016.

[8] G. A. Susto and M. Krstic, "Control of PDE-ODE cascades with Neumann interconnections," Journal of the Franklin Institute, vol. 347, no. 1, pp. 284-314, 2010.

[9] S. Tang and C. Xie, "State and output feedback boundary control for a coupled PDE-ODE system," Systems \& Control Letters, vol. 60, no. 8, pp. 540-545, 2011.

[10] O. M. Aamo, "Disturbance rejection in $2 \times 2$ linear hyperbolic systems," IEEE Transactions on Automatic Control, vol. 58, no. 5 , pp. 1095-1106, 2013.

[11] A. Hasan, O. M. Aamo, and M. Krstic, "Boundary observer design for hyperbolic PDE-ODE cascade systems," Automatica, vol. 68, pp. 75-86, 2016.

[12] F. Di Meglio, F. Bribiesca Argomedo, L. Hu, and M. Krstic, "Stabilization of coupled linear heterodirectional hyperbolic PDE-ODE systems," Automatica, to appear.

[13] F. Castillo, E. Witrant, C. Prieur, and L. Dugard, "Boundary observers for linear and quasi-linear hyperbolic systems with application to flow control," Automatica, vol. 49, no. 11, pp. 3180-3188, 2013.

[14] R. Kress, Linear integral equations, ser. Applied Mathematical Sciences. Springer-Verlag, New York,, 1999, vol. 82. 